\title{
Stringlike Clusters and Cooperative Interlayer Permeation in Smectic Liquid Crystals Formed by Colloidal Rods
}

\author{
Alessandro Patti, ${ }^{1}$ Djamel El Masri, ${ }^{1}$ René van Roij, ${ }^{2}$ and Marjolein Dijkstra ${ }^{1}$ \\ ${ }^{1}$ Soft Condensed Matter, Debye Institute for NanoMaterials Science, Utrecht University, \\ Princetonplein 5, 3584 CC, Utrecht, The Netherlands \\ ${ }^{2}$ Institute for Theoretical Physics, Utrecht University, Leuvenlaan 4, 3584 CE, Utrecht, The Netherlands
}

(Received 16 June 2009; published 9 December 2009)

\begin{abstract}
Using simulations of hard rods in smectic-A states, we find stringlike clusters of up to 10 interlayer rods exhibiting dynamic cooperativity. We also find non-Gaussian diffusion and heterogeneous dynamics due to the equilibrium periodic smectic density profiles, which give rise to permanent barriers for layer-tolayer diffusion. This relaxation behavior is surprisingly similar to that of nonequilibrium supercooled liquids, although there the particles are trapped in transient (instead of permanent) cages.
\end{abstract}

Smectic liquid crystals (LCs) consist of stacks of fluidlike layers of oriented rodlike particles [1]. They can be stabilized by attractive Van der Waals forces [1] or by repulsive hard-core interactions between sufficiently elongated particles [2]. In the smectic- $A$ phase, the static structure is characterized by long-range orientational ordering of the rods combined with a one-dimensional periodic density variation in the direction parallel to the rods. While the equilibrium properties of smectic phases are relatively well understood [1], little is known about their dynamics on the particle scale, even though Helfrich's early report of diffusion ("permeation") of anisotropic particles through smectic layers goes back 40 years [3]. Recently, however, exciting progress was made based on newly developed experimental techniques (e.g., NMR coupled to strong magnetic field gradients [4], or fluorescent labelling of rods [5]), which revealed direct observations of non-Gaussian diffusion and quasiquantized layerto-layer hopping across a barrier [5]. This triggered new theoretical work, based on dynamic density functional theory (DDFT), which not only confirmed the nonGaussian diffusive motion and the one-dimensional "permanent" barriers due to the static smectic background, but also showed the importance of "temporary" cages formed by neighboring rods [6].

Non-Gaussian diffusive behavior due to heterogeneous dynamics of "slow" and "fast" particles is also a key feature in glassy systems and supercooled liquids, in which individual particles are trapped in transient cages formed by their neighbors. This heterogeneous dynamics is found to be closely related to cage rearrangements and to cooperative motion, in which a small fraction of the particles (typically a few percent) move collectively in stringlike $[7,8]$ or compact $[9,10]$ clusters. The intriguing question that we address in this Letter is to what extent the dynamics in the smectic- $A$ phase is collective, or, in other words, to what extent is the equilibrium smectic- $A$ dynamics resemblant to that of out-of-equilibrium quenched supercooled liquids? This issue cannot be addressed directly in fluorescence experiments, in which only a small fraction of the rods is labeled such that moving clusters cannot be observed. DDFT yields average quantities such as density profiles, Van Hove functions, and single-particle barriers, but does not provide cluster information [6]. We therefore resort to computer simulations of hard-rod fluids in the smectic phase. The key observation of this Letter is that we find clear evidence for cooperative permeation of stringlike clusters containing up to 10 fast-moving rods in an equilibrium smectic LC phase.

Our system contains $N=1530-3000$ freely rotating hard spherocylinders with aspect ratio $L^{*} \equiv L / D=5$, where $D$ is the diameter and $L+D$ the rod length. For $L^{*}=5$, the smectic phase melts into a nematic phase below $P^{*}=1.4$ and freezes into a crystal above $P^{*}=$ 2.3 , where $P^{*}=P D^{3} / k_{B} T$ is the reduced pressure with $k_{B}$ Boltzmann's constant [11]. We study the bulk smectic phase at $P_{1}^{*}=1.6$ and $P_{2}^{*}=2.0$, corresponding to packing fractions $\eta_{1}=0.508$ and $\eta_{2}=0.557$, respectively. We performed MC simulations in a rectangular box with 5-10 smectic layers and periodic boundaries. First, we equilibrated the systems at constant $N, P$, and $T$. Then, we performed production runs at constant volume to analyze the relaxation dynamics. Standard MC simulations with small displacements are used to mimic Brownian motion. This was shown to be efficient to study the slow relaxation of glasses at low temperatures [12] or at high concentrations [13]. The anisotropy of the self-diffusion of the rods (a factor of 2 [14]) is taken into account by a larger maximum displacement parallel to the long axis compared to that along the short axes. Translational and rotational moves were selected randomly, with the same probability to attempt each of them separately or together. The acceptance rates of both moves were $\approx 50 \%$. As unit of time, we chose $\tau \equiv D^{2} / D_{\text {tr }}$, where $D_{\text {tr }}$ is the isotropic average of the translational diffusion coefficients in the three space dimensions at short time. We checked that our results (mea- 
sured in units of $\tau$ ) are independent of the maximum displacements.

Denoting the positions of the rods by $\mathbf{r}_{i}=\left(x_{i}, y_{i}, z_{i}\right)$, we first measure the (relative) probability $\pi(z)$ of finding a rod at position $z$, where the $z$ axis is parallel to the nematic director $\hat{n}$. Following Ref. [5], we introduce the Boltzmann factor $\pi(z) \propto \exp \left[-U(z) / k_{B} T\right]$ with $U(z)$ the effective potential for diffusion out of the middle of a smectic layer. The layer spacings in the two states, $h=(L+D) \times$ $(1.05,1.03)$, follow from fits to the periodicity, and the potential barriers are found to be $U_{0}=(3.5,7.5) k_{B} T$. Thus, the denser state reveals a slightly smaller layer spacing but a substantially higher diffusion barrier and hence a much stronger confinement to the middle of the smectic layers. Note that the small size of our system and the periodic boundary conditions prevent the formation of screw disclinations, which could have allowed for barrierfree nematiclike "fast tracks" through the layers [15].

We thus expect heterogeneous dynamics due to the rods rattling around in a given layer until they overcome the barrier and jump to a neighboring layer. To quantify the picture of a rattling and hopping-type $z$ diffusion, we calculate the self part of the Van Hove correlation function (VHF) $\quad G_{s}(z, t)=\frac{1}{N}\left\langle\sum_{i=1}^{N} \delta\left\{z-\left[z_{i}\left(t_{0}+t\right)-z_{i}\left(t_{0}\right)\right]\right\}\right\rangle$, with $\langle\ldots\rangle$ the ensemble average over all particles and initial time $t_{0}$, and $\delta$ the Dirac-delta. Note that $G_{s}(z, t)$ gives the distribution for the particle $z$ displacements during a time interval $t$, and would be a Gaussian of $z$ for freely diffusive particles. Figures 1(a) and 1(b) show the VHF for our two state points as a function of $z$ for several equidistant $t$ 's, showing the appearance of peaks at integer layer spacings consistent with earlier experimental [5] and theoretical [6] results. The height and spatial extension of the peaks is larger at $\eta_{1}$, indicative of a faster layer-to-layer diffusion than at $\eta_{2}$. The VHF's can be analyzed in terms of the non-Gaussian parameter (NGP) [16], for $z$ diffusion defined by $\alpha_{z}(t)=-1+\left\langle\Delta z(t)^{4}\right\rangle / 3\left\langle\Delta z(t)^{2}\right\rangle^{2}$ with $\Delta z(t)=z\left(t_{0}+t\right)-z\left(t_{0}\right)$ the $z$ displacement of a rod in the time interval $t$ starting at $t_{0}$. Likewise, non-Gaussian inplane diffusion can be characterized by an NGP $\alpha_{x y}(t)$. Heterogeneous dynamics occurs on a time-scale $t$ if $\alpha_{z}(t)$ or $\alpha_{x y}(t)$ is nonvanishing. In Figs. 1(c) and 1(d), we show the NGP's for our two state points, with $\alpha_{z}(t)$ showing a clear peak at $t^{*} \simeq(2,10) \tau$ with $\alpha_{z}\left(t^{*}\right)$ increasing with density, while $\alpha_{x y}(t)$ is hardly visible on the scale of the figure and thus essentially vanishes for all $t$. We also plot the mean-square displacements (MSDs) $\left\langle\Delta z^{2}(t)\right\rangle$ and $\left\langle\Delta x^{2}(t)+\Delta y^{2}(t)\right\rangle$ in Figs. 1(e) and 1(f) for the two state points. The $x y$-MSD shows a smooth crossover from shortto long-time diffusion, while the $z$-MSD develops an intermediate cage-trapping plateau up to $t^{*}$ beyond which interlayer diffusion takes place. Note that the $z$-MSD exceeds the $x y$-MSD only at short times due to anisotropy of the short-time diffusion coefficients [14].

In addition, we study structural relaxation by computing the self-intermediate scattering function $F_{s}(t)=\langle\exp [i \mathbf{q} \cdot$
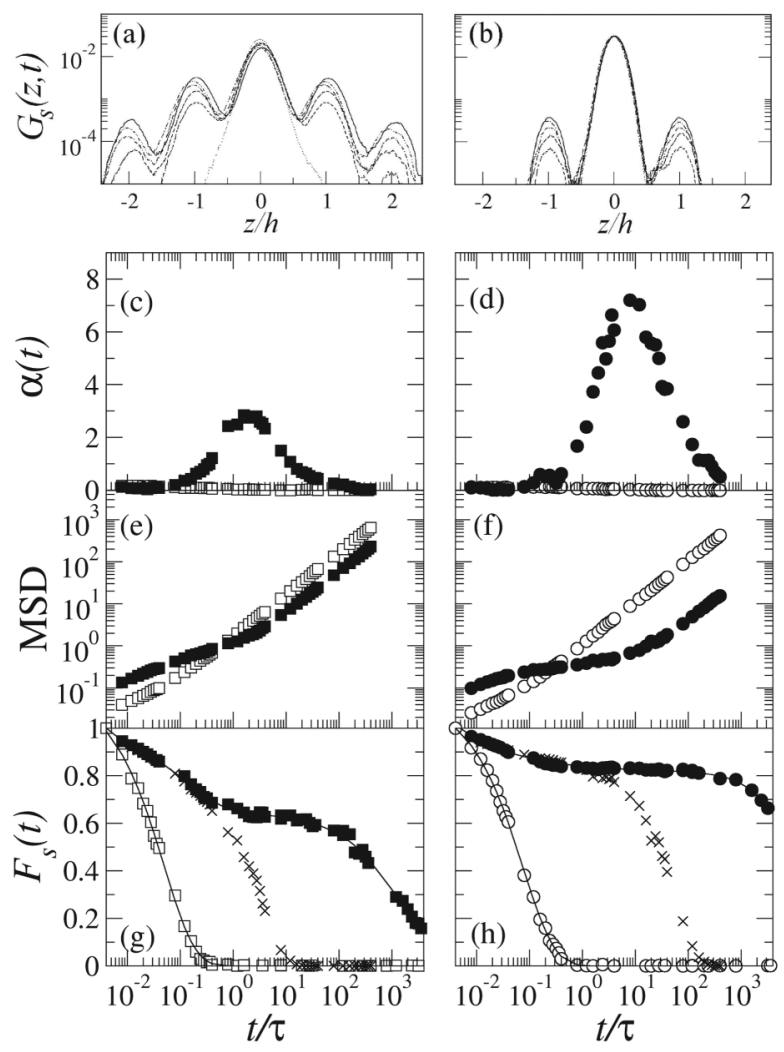

FIG. 1. (a), (b) Self part of the Van Hove function $G_{s}(z, t)$; (c), (d) non-Gaussian parameter $\alpha_{z}(t)$ and $\alpha_{x y}(t)$; (e), (f) meansquare displacement (MSD) in units of $D^{2}$; and $(\mathrm{g})$, (h) $F_{s}(t)$ and its Gaussian approximation (crosses, see text), at packing fraction $\eta_{1}=0.508$ (left column) and $\eta_{2}=0.557$ (right column). The time intervals in (a), (b) range from $t=0.4 \tau$ (dotted lines) to $t=40 \tau$ (solid lines), with increments of $\approx 8 \tau$. The solid and open symbols refer to the $z$ and $x y$ direction, respectively.

$\Delta \mathbf{r}(t)]\rangle$ at wave vectors $\mathbf{q} D=\left(0,0, q_{z}\right)$ and $\left(q_{x}, q_{y}, 0\right)$, with $q_{z}=1$ and $\left(q_{x}^{2}+q_{y}^{2}\right)^{1 / 2}=6$, that correspond to the main peaks in the static structure factor. Here $\Delta \mathbf{r}(t)$ denotes the particle displacement during a time interval $t$. Results are shown in Figs. 1(g) and 1(h). The in-layer dynamics is very fast with slightly stretched exponential decay, typical of dense fluidlike behavior [17]. By contrast, the interlayer dynamics is much slower, with $F_{s}(t)$ decaying in two steps at $t^{\prime}$ and $t^{\prime \prime}$ separated by a plateau during $t^{\prime}<t<t^{\prime \prime}$. For comparison, we also plot the Gaussian approximation $F_{s}^{G}(t)=\exp \left[-q_{z}^{2}\left\langle\Delta z^{2}(t)\right\rangle\right]$, which lacks a clear plateau. We conclude that the plateau coincides with the nonvanishing of $\alpha_{z}(t)$, and can hence be attributed to the heterogeneity of the dynamics [7]. The first step at $t^{\prime} / \tau \leq 1$ corresponds to the rattling of rods inside the temporary cage and permanent smectic background formed by neighboring rods [6], whereas the second one ( $\alpha$ relaxation) corresponds to the escape on a time scale that increases from $t^{\prime \prime} / \tau \simeq 100$ at $\eta_{1}$ to $10^{3}$ at $\eta_{2}$. The increase of $t^{\prime \prime}$ and that of the height of the plateau of $F_{s}(t)$ with density is also observed for colloidal glasses [17]. At $\eta_{1}$, the longtime decay of $F_{s}(t)$ is well fitted by a stretched exponential of 
the form $\exp \left[-\left(t / t_{r}\right)^{\beta}\right]$, with relaxation time $t_{r} / \tau \cong 650$ and $\beta \cong 0.6$, once more confirming the heterogeneous nature of the relaxation dynamics [17]; $t_{r}$ at $\eta_{2}$ is beyond our simulation time.

Non-Gaussian dynamics due to rattling-and-jumping processes are not surprising, and have in fact been observed in, e.g., 2D liquids [18], cluster crystals [19], and glasses [20]. Several models based on a heterogeneous distribution of diffusion coefficients, jump times, or jump lengths have been introduced to explain the heterogeneous dynamics due to temporary cages [20], while another model explains the non-Gaussian diffusion by the dynamics of a single Brownian particle in a periodic external potential, which is very similar to the permanent barriers of the smectic- $A$ phase [21]. The new feature that we identify below concerns the cooperative motion of strings of fastmoving particles. This spatial structure and cooperativity cannot be captured by the essentially one-particle analyses of Refs. [21,22], has not been observed in Refs. [18,19], but yields an intriguing analogy with glassy dynamics. It is also tempting to speculate that the cooperative motion of strings of different sizes might be responsible for a distribution of decay rates, and hence for the stretchedexponential decay of $F_{s}(t)$.

To this end, we first identify the fast-moving particles. Comparing the VHF at $t^{*}$ of Figs. 1(a) and 1(b) with a Gaussian approximation with the same MSD reveals that the fast-moving particles have travelled over more than $h / 2$ during a time interval $t^{*}$. Therefore, they are intimately related to interlayer particles, which reside more than some distance $\delta$ from the nearest smectic plane. In order to define $\delta$ sensibly, we consider the variance of one period of $\pi(z)$ as $\sigma^{2} \equiv \int_{-h / 2}^{h / 2} z^{2} \pi(z) d z$, which gives $\sigma / D=$ $(0.6,0.3)$ for $\eta_{1}$ and $\eta_{2}$, respectively. We now set $\delta=$ $k \sigma$ with $k=1,2,3$. The fraction $f_{k}$ of so-called interlayer particles is then $f_{1}=(0.28,0.45), f_{2}=(0.05,0.13)$, and $f_{3}=(0.02,0.03)$ for our two state points, showing, perhaps surprisingly, that the denser state contains more interlayer particles caused by the smaller $\sigma$. We also calculated the pair distribution of the interlayer particles (not shown), revealing a higher contact value than that of the bulk smectic phase, suggesting substantial clustering of interlayer particles despite their low concentration $f_{k} \eta$. Stringlike clusters composed of $n=1, \cdots, 10$ interlayer rods can indeed be identified, as shown for $\delta=2 \sigma$ by the size distribution $P(n)$ in Fig. 2. Our (rather stringent) cluster criterion is such that two interlayer particles belong to the same cluster if the $z$ and $x y$ distances are smaller than $h$ and $D$, respectively. At $\eta_{1}$, one deduces from $P(n)$ that $\approx 95 \%$ of the clusters consists mostly of 2 or 3 rods, while clusters of more than 5 rods are rare but do exist. The denser smectic phase has larger clusters, which is again similar to supercooled liquids and glassy systems, in which the cluster size increases with increasing cage trapping $[8,10]$. The fit $P(n) \propto \exp (-\alpha n)$ is accurate with $\alpha=$ $(1.5,0.7)$ at $\eta_{1}$ and $\eta_{2}$, respectively, from which the aver-
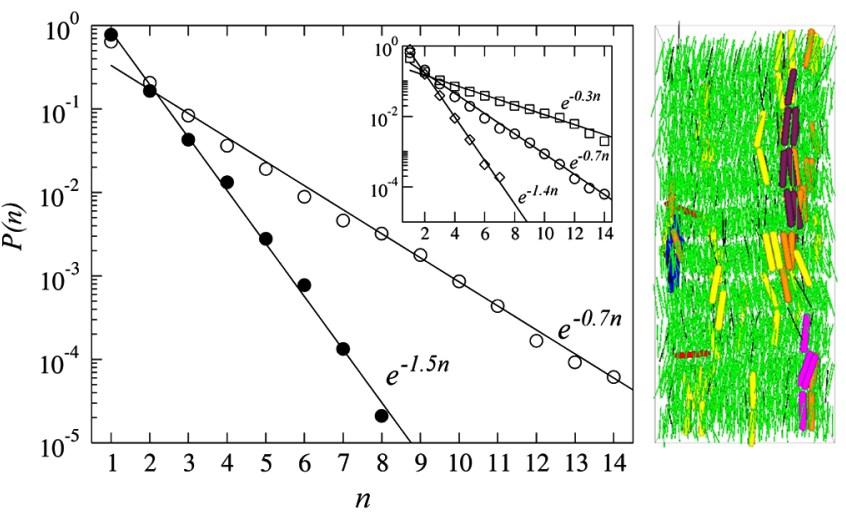

FIG. 2 (color online). Probability distribution $P(n)$ of the number $n$ of interlayer rods in a stringlike cluster (with $\delta=$ $2 \sigma$, see text), at $\eta_{1}(\bigcirc)$ and $\eta_{2}(\bigcirc)$. The inset shows $P(n)$ at $\eta_{2}$ for $\delta=\sigma(\square), 2 \sigma(\bigcirc)$, and $3 \sigma(\diamond)$. The solid lines denote the fit $P(n) \propto \exp (-\alpha n)$ with $\alpha$ given in the figure. The snapshot of 3000 rods at $\eta_{1}$ shows predominantly inlayer rods and single interlayer rods (black), both with diameters reduced to $D / 4$ for clarity. The thicker rods denote transverse ones as well as stringlike clusters of 2 (light shaded) up to 7 (dark shaded) rods.

age cluster size follows as $\langle n\rangle=[1-\exp (-\alpha)]^{-1}$. These results, however, depend strongly on $\delta$ as revealed by the inset of Fig. 2.

With all these resemblances to glassy dynamics, we now study the dynamics of the layer-to-layer diffusion, for which Fig. 3(b) shows some typical trajectories. Most of the rods "jump" fast compared to the dwelling time within a smectic layer. Some rods diffuse to the interlayer spacing and return to their original layer; others move from one layer to another several times; in some cases, the dwelling time in the interlayer spacing is quite long, while double jumps can be also observed. These observations suggest a rather broad distribution of jump times $t_{J}$ to diffuse from one layer to the next, where $t_{J}$ is defined as the time span between first and last "contact" with the new and old layer, respectively, with contact established if the rod is at a
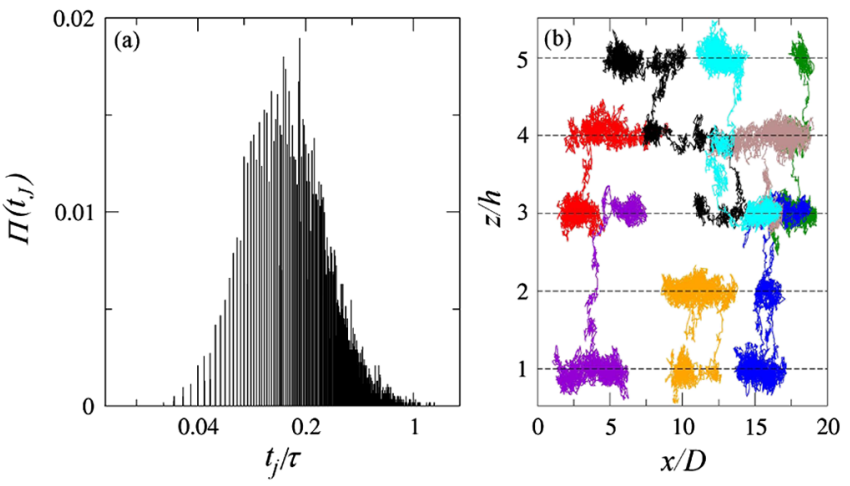

FIG. 3 (color online). (a) Distribution $\Pi\left(t_{J}\right)$ of layer-to-layer jump times $t_{J}$, based on $\delta=2 \sigma$ at density $\eta_{1}$. (b) Trajectories of jumping rods projected onto the $x z$ plane, with the dashed lines representing the middle of the smectic layers. 
distance $\delta$ from the middle of a smectic layer. At $\eta_{1}$ and for $\delta=2 \sigma$, the distribution of jump times $\Pi\left(t_{J}\right)$ as obtained by averaging over many trajectories is plotted in Fig. 3(a), and shows that the most probable jump time is $0.14 \tau$, the median is at $t_{J}^{*}=0.2 \tau$, while the distribution extends over two decades $0.01<t_{J} / \tau<1$. Having developed a sense of time, we can characterize the trajectories further. We distinguish single and multiple jumps, depending on the dwelling time in the next layer being longer or shorter, respectively, than $t_{J}^{*}$ before another jump is started. At $\eta_{1}$, we find that $\approx 5 \%$ of the total number of jumps is multiple, of which $\approx 83 \%$ is a double and the remaining fraction a triple jump. At $\eta_{2}$, where $t_{J}^{*}=0.27 \tau$, the fraction of multiple jumps is $0.6 \%$. The interlayer rods are generally oriented along $\hat{n}$. Remarkably, however, at $\eta_{1}$ also transversely oriented rods in between two smectic layers have occasionally been observed [23,24], which diffuse either to a new layer or return to the original one by rotating parallel or antiparallel to their original orientation.

With our clear evidence for heterogeneous dynamics and spatial correlations between the fast-moving interlayer particles, it seems natural to investigate dynamic cooperativity, i.e., whether or not the stringlike clusters observed in static configurations actually move collectively. This requires a cluster criterion that not only involves spatial but also temporal proximity to identify collectively moving rods. Two jumping rods $i$ and $j$ are considered to move cooperatively if their arrival times $t^{(i)}$ and $t^{(j)}$ in their new layers (i.e., the first time at which their distance to the middle of the new layer equals $\delta$ ) satisfies $\left|t^{(i)}-t^{(j)}\right|<\Delta t$, while $\mathbf{r}_{i}\left(t^{(i)}\right)$ and $\mathbf{r}_{j}\left(t^{(j)}\right)$ satisfy our static spatial cluster criterion. Using $\delta=2 \sigma$ and $\Delta t=\tau$ [i.e., long enough for any jump to finish according to $\left.\Pi\left(t_{J}\right)\right]$, we find at $\eta_{1}$ that the number of collective jumps is $\approx 25 \%$ of the total number of jumps, and involves mainly $2(\approx 79 \%)$ or $3(\approx 17 \%)$, and very rarely $\geq 4(<4 \%)$ rods. Interestingly, $\approx 42 \%$ of the collective jumps involve rods diffusing in opposite directions, suggesting that the vacated space of a jumping rod is reoccupied by a rod from the two neighboring layers with almost equal probability. These characteristic values are insensitive to small modifications of our spatial cluster criterion, while a smaller temporal interval of $\Delta t=t_{J}^{*}$ (the median jump time) reduces the fraction of collective jumps to $\approx 9 \%$. In other words, the motion is indeed strongly cooperative at $\eta_{1}$. If the analogy with glassy systems would hold even further, one would expect more cooperativity at $\eta_{2}$. Surprisingly, perhaps, despite the larger static clusters, we find less collective motion at $\eta_{2}$. For instance, with $\delta=2 \sigma$ and $\Delta t=1.2 \tau$ (maximal jump time), only $\approx 4 \%$ of the jumps can now be regarded as collective (involving essentially only 2 rods). We attribute this reduction of cooperativity upon approaching crystallization to the higher permanent barriers that reduce the probability of static clusters to actually complete their attempted jumps. Such permanent barriers do not exist in undercooled liquids (where the dynamic cluster size grows upon approaching the glass transition $[8,10])$, whereas they do exist in crystals, where structural relaxation is virtually nonexistent.

In conclusion, for equilibrium hard rods in the smectic phase, we find non-Gaussian dynamics due to the periodic smectic structure and cooperative motion of stringlike clusters surprisingly similar to that of nonequilibrium supercooled liquids. Our results might be relevant for dynamics in other inhomogeneous liquids, e.g., confined fluids (micro and nanofluidic devices) [25], columnar LCs, and cellular processes such as cell death and signaling in lipid membranes [26].

We thank P. van der Schoot and M. Bier for useful discussions. This work was financed by a NWO-VICI grant.

[1] P. G. de Gennes and J. Prost, The Physics of Liquid Crystals (Clarendon, Oxford, 1993).

[2] D. Frenkel et al., Nature (London) 332, 822 (1988).

[3] W. Helfrich, Phys. Rev. Lett. 23, 372 (1969).

[4] I. Furó and S. V. Dvinskikh, Magn. Reson. Chem. 40, S3 (2002).

[5] M. P. Lettinga and E. Grelet, Phys. Rev. Lett. 99, 197802 (2007).

[6] M. Bier et al., Phys. Rev. Lett. 101, 215901 (2008).

[7] W. Kob et al., Phys. Rev. Lett. 79, 2827 (1997).

[8] C. Donati et al., Phys. Rev. Lett. 80, 2338 (1998).

[9] G. A. Appignanesi et al., Phys. Rev. Lett. 96, 057801 (2006).

[10] E. R. Weeks et al., Science 287, 627 (2000); L. Berthier et al., Science 310, 1797 (2005).

[11] P. Bolhuis and D. Frenkel, J. Chem. Phys. 106, 666 (1997).

[12] L. Berthier and W. Kob, J. Phys. Condens. Matter 19, 205130 (2007).

[13] L. Pfleiderer et al., Europhys. Lett. 84, 16003 (2008).

[14] M. Doi and S.F. Edwards, The Theory of Polymer Dynamics (Clarendon Press, Oxford, 1994).

[15] R. L. Selinger, Phys. Rev. E 65, 051702 (2002).

[16] A. Rahman, Phys. Rev. 136, A405 (1964).

[17] G. Brambilla et al., Phys. Rev. Lett. 102, 085703 (2009).

[18] M. M. Hurley and P. Harrowell, J. Chem. Phys. 105, 10521 (1996).

[19] A. J. Moreno and C. N. Likos, Phys. Rev. Lett. 99, 107801 (2007).

[20] P. Chaudhuri et al., Phys. Rev. Lett. 99, 060604 (2007).

[21] B. Vorselaars et al., Phys. Rev. E 75, 011504 (2007).

[22] G. Cinacchi and L. De Gaetani, Phys. Rev. E 79, 011706 (2009).

[23] R. van Roij et al., Phys. Rev. E 52, R1277 (1995).

[24] J. S. van Duijneveldt and M. P. Allen, Mol. Phys. 90, 243 (1997).

[25] J. Mittal et al., Phys. Rev. Lett. 100, 145901 (2008); C. R. Nugent et al., Phys. Rev. Lett. 99, 025702 (2007).

[26] E. Falck et al., J. Am. Chem. Soc. 130, 44 (2008). 